\title{
CUIDADOS PALIATIVOS EM INSTITUIÇÃO DE LONGA PERMANÊNCIA FRENTE À PANDEMIA DA COVID-19: REFLEXÕES A PARTIR DA TEORIA DO FINAL DE VIDA PACÍFICO
}

Tânia Maria de Oliva Menezes' ORCID: 0000-0001-5819-0570

Valéria Moreira da Silva" ORCID: 0000-0001-6786-6325

Daniel Espírito Santo da Silva"I ORCID: 0000-0001-9011-9571

Universidade Federal da Bahia. Salvador, Bahia, Brasil.

" Universidade Federal de Rondônia. Porto Velho, Rondônia, Brasil.

III Universidade Federal Fluminense. Rio de Janeiro, Rio de Janeiro, Brasil.

Autor Correspondente: Tânia Maria de Oliva Menezes E-mail: tomenezes50@gmail.com

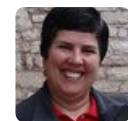

Como citar

Menezes TMO, Silva VM, Silva DES. Cuidados paliativos em instituição de longa permanência frente à pandemia da covid-19: reflexões a partir da teoria do final de vida pacífico. In: Santana RF. Enfermagem gerontologica no cuidado do idoso em tempos da COVID 19. 2.ed.rev. Brasilia, DF: Editora ABEn; 2020. p.29-34. (Serie Enfermagem e Pandemias, 1). https://doi.org/10.51234/aben.20.e01.c05

\section{INTRODUÇÃO}

O novo coronavírus, nomeado como SARS-CoV-2, tem alta transmissibilidade e provoca uma síndrome respiratória aguda (COVID-19), que varia de casos leves, cerca de $80 \%$, a casos muito graves, com insuficiência respiratória, entre $5 \%$ e $10 \%$ dos casos, além de alta letalidade em idosos, principalmente acima de 80 anos ${ }^{(1)}$.

Diante do contexto atual da pandemia de COVID-19, reconhecida pela Organização Mundial de Saúde, e o agravamento de sintomas conhecidos até o momento, além do risco que envolve principalmente para a população idosa, se faz necessário compartilhar conhecimentos pertinentes à prática de Cuidados Paliativos nas Instituições de Longa Permanência para Idosos (ILPIs). O Cuidado paliativo fornece suporte ideal aos pacientes frente aos sintomas angustiantes, tais como dor, dificuldades respiratórias, náusea, medo ou confusão e promove também medidas de reabilitação, diagnóstico e terapêuticas que possam contribuir para a qualidade de vida do paciente ${ }^{(2)}$.

Para subsidiar a prática dos $\mathrm{CP}$ a idosos que vivem nas ILPIs frente ao COVID-19, optou-se pela Teoria do Final de Vida Pacífico, apresentada pelas enfermeiras Rouland e Moore, que propõe o alívio dos medos e ansiedade, reais e/ou percebidos, para o paciente e sua família ${ }^{(3)}$.É inegável a importância da Teoria do Final de Vida Pacífico para subsidiar a prática do enfermeiro à luz dos cuidados $\mathrm{CP}^{(4)}$.

Temas como manejo de sintomas que ameacem a qualidade de vida, diretivas antecipadas de vontade, comunicação de más notícias são fundamentais nessa filosofia de cuidado, e se tornam imprescindíveis nesse contexto da Pandemia. Conhecer as ferramentas que temos à nossa disposição e nos prepararmos para participar de momentos difíceis, com o máximo de conhecimento sobre intervenções possíveis. Este conhecimento tornará mais suave as decisões difíceis que precisarão ser tomadas. 
O Departamento Científico de Enfermagem Gerontológica, da Associação Brasileira de Enfermagem, diante do quadro de pandemia, com agravos para ILPI, apresenta diretrizes para o cuidado de idosos em paliação nestas instituições.

\section{OBJETIVO}

Discutir o cuidado ao idoso em paliação que reside em instituição de longa permanência frente a infecção por coronavírus a partir da Teoria do Final de Vida Pacífico.

\section{MÉTODO}

Ensaio teórico de natureza reflexiva, baseado na TFVP, portarias e artigos científicos nacionais e internacionais.

\section{RESULTADOS}

Medidas de cuidados para aliviar os idosos em paliação nas ILPI, embasado nos conceitos TFVP, devem ser observadas: Conforto, não sentir dor, proporcionar respeito e dignidade, estar em paz e ficar próximo a pessoas significativas, conforme a figura 1.

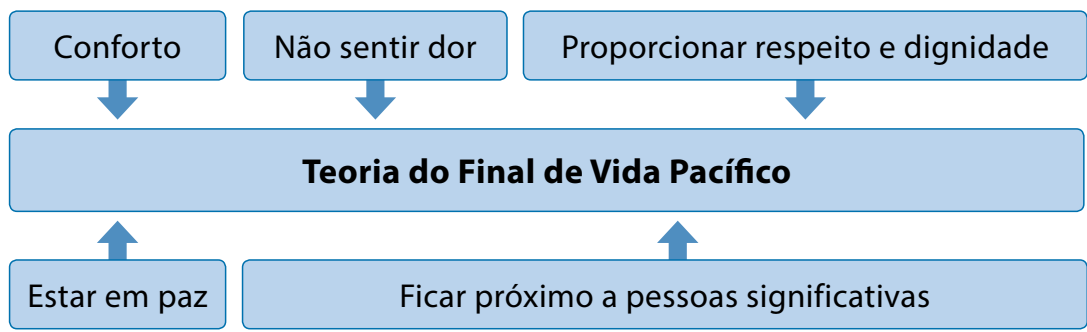

Figura 1: Propostas da Teoria do Final de Vida Pacífico para a qualidade de vida do paciente no final da vida

\section{Conforto}

A Teoria do Final de Vida Pacífico apresenta a experiência de conforto como tudo o que torna a vida fácil e agradável na vida ${ }^{(3)}$. O alívio de todo tipo de desconforto deve ser prioridade no cuidado. As intervenções da equipe de enfermagem tornam-se parte integrante do atendimento aos idosos em paliação nas ILPIs, as quais são consideradas boas práticas de enfermagem na promoção de higiene e conforto:

- Estimular o relaxamento, através de música ou diálogo;

- Higiene corporal e massagem de conforto;

- Posicionamento no leito/cama;

- Manter os ambientes ventilados naturalmente (janelas abertas), higienizados, livres de ruídos, odores e iluminação excessiva.

O conforto manifesta-se por sutilezas da presença do profissional, ao dedicar um pouco mais de atenção, ficar ao lado do idoso, olhar nos seus olhos, sorriso, palavras de consolo, e até no silêncio ${ }^{(5)}$. O conforto espiritual também deve ser pensado ao cuidar do idoso em paliação nas ILPI. Na prática, a atenção do profissional está mais direcionada aos aspectos físicos. 
Atentar-se para o desconforto respiratório, sintoma comum nos casos de COVID-19, o qual pode ser aliviado com a elevação da cabeceira do leito em $45^{\circ}$, relaxamento dos músculos acessórios a respiração. Verificar saturação de oxigênio (SpO2) e se menor que 95\% em ar ambiente ${ }^{(1)}$, oferecer suporte de oxigenioterapia (consultar protocolo do serviço). Atenção a possível ocorrência de taquipneia, considerada quando a frequência respiratória é maior que 24 respirações por minuto $(F R>24 \mathrm{rpm})^{(1)}$.

No cuidado paliativo, o objetivo de conforto prevalece também na escolha da via de administração dos fármacos. Na medida do possível, recomenda-se simplificar a prescrição e evitar vias endovenosas que agregam desconforto, dependência, riscos e custos geralmente desnecessários. No entanto, os idosos com Síndrome Respiratória pelo Novo Coronavírus (SARS-COV-2) é preconizado o uso de terapêutica medicamentosa por via intravenosa, de acordo com o protocolo de Manejo Clínico da COVID-19 na Atenção Especializada ${ }^{(6)}$.

\section{Não sentir dor}

O manejo eficaz da dor reduz o desconforto físico, todavia, por ser um dado subjetivo é necessário uma avaliação minuciosa por meio de instrumentos específicos, como escalas numéricas que classificam a dor, de 0 a 10, com 0 indicando ausência e 10, dor muito intensa, escala visuais/fácies e ou descritivas ${ }^{(7)}$. As escalas não são instrumentos diagnósticos, é uma ferramenta de auxílio ou de screening. Algumas escalas foram desenvolvidas para avaliação de dor em idosos com demência, como por exemplo, a PACSLAC (Pain Assessment Checklist for Seniors with Limited Ability to Communicate) validada na versão brasileira no estudo desenvolvido em uma casa de repouso, com idosos que apresentavam comunicação verbal limitada, quando expostos a situações potencialmente dolorosas ${ }^{(8)}$.

A avaliação deve ser realizada pelos profissionais de enfermagem das ILPI's, que por meio da avaliação diária identificam a intensidade e o tipo de dor. Importante atentar-se para idosos com déficits cognitivos, sensoriais e que impossibilitados de expressarem verbalmente, manifestam sinais de dor por meio de movimentos como dentes cerrados, colocar as mãos sobre a área de dor, posturas curvadas, fácies de dor, gemidos e agitação(7).

O plano de cuidados para a promoção do alívio e controle da dor deve incluir medidas farmacológicas (protocolo da Instituição), e intervenções não farmacológicas, dentre as quais se destacam:

- Aplicação de calor ou frio;

- Compressas geladas;

- Adotar posição de conforto (alinhamento corporal), com apoio de coxins para alívio da pressão nas articulações;

- Exercícios de relaxamento;

- Controle de estímulos ambientais que possam influenciar a dor;

- Cuidado espiritual.

Nas práticas de cuidado ao idoso em paliação nas ILPIs é preciso ainda considerar outros aspectos da dor: social, psíquica, financeira e espiritual, que se manifestam devido à:

- Dificuldade de aceitação da morte;

- Medo do sofrimento e morte;

- Perda do sentido da vida com a iminência da morte;

- Afastamento de familiares e pessoas significativas;

- Distanciamento de práticas que o aproximavam de sua espiritualidade.

A terapia Relaxamento, Imagens Mentais e Espiritualidade (RIME), que integra técnicas de relaxamento mental e visualização de imagens com elementos que representam a espiritualidade, ainda pouco conhecida 
em nosso meio, se apresenta como uma das possibilidades de ressignificar a dor não física e melhorar a qualidade de vida de idosos em paliação nessas instituições.

\section{Proporcionar respeito e dignidade}

A experiência da dignidade é definida como um atributo importante dentro da teoria, que envolve ser reconhecido e respeitado como igual e não ser exposto a qualquer situação que viole sua integridade ou valores ${ }^{(3)}$. $O$ idoso no cenário das ILPIs é um ser humano que deve ser estimulado diariamente quanto a manutenção de sua autonomia e independência, portanto, com vontades respeitadas, direito de defesa, mesmo quando dependente ${ }^{(4)}$.

Para tratar um idoso nesse cenário com dignidade é necessário incluí-lo nas escolhas que serão tomadas, assim como com suas famílias. Essas decisões compartilhadas devem ser feitas utilizando empatia e respeito ${ }^{(9)}$ e são essenciais para a qualidade de vida e, eventual terminalidade da pessoa idosa.

As diretivas antecipadas de vontade dos idosos devem ser respeitadas. Conhecer ao máximo possível a biografia das pessoas cuidadas, já deveria ser algo discutido anteriormente, mas a situação atual tornou urgente sua compreensão, uma vez que passamos repentinamente a ter várias discussões relacionadas ao tema, sempre à luz da ética.

O respeito e dignidade que se pode oferecer ao idoso nas ILPIs consistem em:

- Cuidar do idoso, até o fim, como ser humano;

- Permitir que o idoso conduza, dentro do possível, o processo que vive, tornando-o participativo em seu processo de cuidado;

- Comunicar tudo que será feito com ele, independente do nível de consciência;

- Oferecer um ambiente acolhedor, livre de ruídos, com iluminação e ventilação adequada;

- Atender suas solicitações, ultrapassando as dificuldades para que suas demandas sejam atendidas;

- Coletar, registrar e respeitar suas diretivas antecipadas.

Esses são alguns exemplos de como aplicar respeito e dignidade aos idosos em paliação nas ILPIs frente a infecção por coronavírus. Recomenda-se que profissionais que tenham maior prática em Cuidados Paliativos sirvam como facilitadores desta condução clínica, considerando que esta será uma abordagem cada vez mais necessária.

\section{Estar em paz}

Para que o idoso em paliação possa estar em paz, o profissional deve proporcionar tranquilidade nos aspectos físicos, psicológicos e espirituais, além de possibilitar que ele não seja incomodado por ansiedade, nervosismo, preocupações e medo ${ }^{(3)}$. Entre suas ações, destacam-se:

- Permitir a expressão de medos, preocupações e ansiedades, principalmente com relação à morte. Promover o diálogo e escuta sensível favorece o estabelecimento de confiança, acolhimento e manifestação do idoso;

- Oportunizar escuta de músicas e programas religiosos nas rádios, assistir programas religiosos na televisão ou leitura de mensagens;

- Apoiar as práticas que favoreçam a reflexão e a expressão de sua fé, tais como, orar, meditar, adorar, ler as escrituras;

- Ambiente tranquilo, livre de ruídos e iluminação excessiva;

- Meditação e o mindfulness, são técnicas utilizadas para focar o pensamento em uma direção. Ao idoso em ILPI, é possível mostrar imagens da natureza. Em seguida, pedir para que feche os olhos e pense na paisagem, conduzindo-o ao silêncio interior, o que possibilita afasta-lo momentaneamente de suas ansiedades, algum desconforto e pensamentos relacionados à morte (5); 
- Possibilitar a presença de pessoas significativas;

- Cuidar com atitudes humanizadas;

- Técnica RIME.

A equipe de enfermagem pode proporcionar paz ao idoso cuidando com respeito e dignidade, atendendo as solicitações que sejam possíveis; identificando as fases de negação e raiva, sem confrontá-lo e acolhendo suas reações ${ }^{(10)}$. Os profissionais que cuidam de idosos em paliação nas ILPIs precisam se aproximar mais da dimensão espiritual, integrando a espiritualidade no seu plano de cuidados, que certamente contribuirá para o idoso estar em paz.

\section{Ficar próximo a pessoas significativas}

Segundo a Teoria do Final de Vida Pacífico permitir a conexão com pessoas que tenham significado é de suma importância. Apesar da sugestão de suspensão de visitas, permitir o contato com pessoas significativas, orientando sobre medidas de prevenção é fundamental no fim de vida. Se o idoso recebia visitas, conversar com a família sobre a possibilidade de alternativas ${ }^{(6)}$.

É necessário preservar a esperança, oferecer acolhimento e suporte, a promoção do contato do idoso com familiares e amigos através dos meios de comunicação, seja por ligações e chamadas de vídeos são fundamentais. Outra alternativa é trazer fotos de familiares e amigos e conversar com o idoso sobre o que significa aquela pessoa em sua vida ${ }^{(6)}$.

Importante considerar que nem todos os idosos que estão em ILPIs ainda mantém vínculo com familiares, tendo em vista que muitos destes desaparecem ao longo do tempo. Entretanto, os idosos transformam alguns profissionais que cuidam em pessoas significativas. É importante reconhecer na instituição quem são essas pessoas significativas para idosos que já não mantém vínculos com familiares e amigos e, de preferência, colocá-las para estar mais próxima deles, diante das possibilidades. Idosos em Cuidados paliativos nas ILPI devem se possível estar em quartos individualizados.

\section{Limitações}

O estudo apresenta como limitações a escassez de artigos para ampliar a discussão, devido à atualidade da temática e cenário de estudo. No entanto o diálogo, destacando questões que envolvem a morte, a escuta sensível e acolhimento são imprescindíveis diante do idoso em final de vida nessas instituições, sendo prioridade nas ações profissionais diante da COVID-19.

\section{Contribuições para Enfermagem}

A reflexão permite uma integração dos princípios ético-científicos do cuidar em enfermagem nas ILPI's e os fundamentos da Teoria do Final de Vida Pacífico no atendimento aos idosos em paliação, favorecendo a dignidade, o respeito e a qualidade do cuidado prestado aos idosos acometidos pela COVID-19. Assim, essa integração possibilita a construção de uma abordagem mais humanitária e significativa diante da Pandemia de infecções por coronavírus.

Nessa perspectiva, as experiências do CP ao idoso que reside nas ILPI's oportunizam aos profissionais de enfermagem uma autoavaliação das habilidades atitudinais e comunicacionais, bem como o fortalecimento do vínculo com os idosos, família e colegas de trabalho.

\section{CONSIDERAÇÕES FINAIS}

Os conceitos apresentados pela Teoria do Final de Vida Pacífico contemplam alguns princípios dos Cuidados Palitativos, podendo subsidiar as ações dos profissionais que cuidam de idosos em paliação que residem nas 
ILPIs frente à Pandemia da COVID-19, tendo em vista que esses necessitam de uma assistência integral que contemple todas as dimensões, de forma a promover uma morte digna e pacífica.

\title{
AGRADECIMENTO
}

\author{
Ao Departamento Científico de Enfermagem Gerontológica da ABEn Nacional.
}

\section{REFERÊNCIAS}

1. Brasil, MS. Secretaria de Atenção Primária à Saúde. Protocolo de Manejo Clínico do Coronavírus (Covid-19) na Atenção Primária à Saúde [Internet] 2020 [Acesso 2020 Abr 15]. Disponível em: https://www.saude.gov.br/images/pdf/2020/ marco/20/20200318-ProtocoloManejo-ver002.pdf

2. Académie Suisse des Sciences Médicales (ASSM). Medical Ethical Guidelines. Palliative Care. Tradução Jeff Acheson, Bottmingen, [Internet] 2017 [Acess 2020 Apr 17]. Available from: https://www.sams.ch/en.html.

3. Ruland CM, Moore SM. Tehory construction based on Standards of care: a proposed theory of the peaceful end of life. Nurs Outlook [Internet] 1998 [cited 2020 Apr 05]; 46(4):69-75. Available from: https://www.sciencedirect.com/science/article/ abs/pii/S0029655498900690

4. Zaccara AAL et al. A analysis and assessment of the peaceful end of life theory according to fawcett's criteria. Texto contexto - enferm [Internet]. 2017 [cited 2020 Apr 19];26(4):e2920017. Available from: http://www.scielo.br/scielo. php?pid=S0104-07072017000400615\&script=sci_abstract

5. Menezes TMO. Diagnósticos e intervenções de enfermagem relacionados à dimensão espiritual aplicados a idosos em cuidados paliativos. In: Associação Brasileira de Enfermagem; Alvarez AM, Caldas CP, Gonçalves LHT, organizadoras. PROENF Programa de Atualização em Enfermagem: Saúde do Idoso. Ciclo 2. Porto Alegre: Artmed Panamericana; 2019. p. 77-105.

6. Brasil, M. Protocolo de manejo clínico da Covid-19 na Atenção Especializada [Internet] 2020 [Acesso 2020 Abr 20]. Disponível em: https://portalarquivos.saude.gov.br/images/pdf/2020/April/14/Protocolo-de-Manejo-Cl--nico-para-o-Covid-19.pdf

7. Eliopoulos C. Enfermagem Gerontológica. Cuidados no Final da Vida. Porto Alegre: Artmed, 9a Ed., p.514-527, 2019.

8. Thé KB, Gazoni FM, Cherpak GL, Lorenzet IC, Santos LA, Nardes EM, Santos FC. Pain assessment in elderly with dementia: Brazilian validation of the PACSLAC scale. Einstein. [Internet]. 2016 [Acesso 2020 Abr 22];14(2):152-7. Available from: http:// www.scielo.br/pdf/eins/v14n2/1679-4508-eins-14-2-0152.pdf.

9. Sandoval S. et al. Muerte y morir en el hospital: una mirada social, espiritual y ética de los estudiantes. Esc. Anna Nery [Internet] 2020 [cited 2020 Apr 19]; 24(3):e20190287, 2020. Available from: https://doi.org/10.1590/2177-9465-ean-2019-0287

10. Menezes TMO. Cuidados de Enfermagem ao Idoso no fim da vida: um olhar a partir da teoria do final de vida pacífico. In: Associação Brasileira de Enfermagem; Alvarez AM, Caldas CP, Gonçalves LHT, organizadoras. PROENF Programa de Atualização em Enfermagem: Saúde do Idoso. Ciclo 1. Porto Alegre: Artmed Panamericana; 2019. p. 141-61. 